\title{
Dispersion and damping of multiple quantum-well polaritons from resonant Brillouin scattering by folded acoustic modes
}

\author{
B. Jusserand,${ }^{1}$ A. Fainstein, ${ }^{2}$ R. Ferreira,${ }^{3}$ S. Majrab,${ }^{1}$ and A. Lemaitre ${ }^{4}$ \\ ${ }^{1}$ Institut des Nanosciences de Paris, CNRS UMR 7588, Université Pierre et Marie Curie (UPMC), \\ F-75005 Paris, France \\ ${ }^{2}$ Instituto Balseiro and Centro atomico Bariloche, Comisión Nacional de Energía Atómica (CNEA), R8402AGP Bariloche, Argentina \\ ${ }^{3}$ Laboratoire Pierre Aigrain, Ecole Normale Supérieure (ENS), CNRS UMR 8551, Université Pierre et Marie Curie (UPMC), \\ Université Paris Diderot (UPD), F-75005 Paris, France \\ ${ }^{4}$ Laboratoire de Photonique et de Nanostructures, CNRS UPR 20, F-91460 Marcoussis, France
}

(Received 14 December 2011; published 11 January 2012)

\begin{abstract}
We demonstrate that resonant Brillouin light scattering using ultranarrow lasers and high-resolution spectrometers provides unique access to the polariton lifetimes, a key parameter in their formation and dynamics. In very high-quality GaAs/AlAs multiquantum wells, we observed significant variations across the resonance of the folded acoustic phonon linewidths, in addition to varying line shifts as reported in bulk materials. Inclusion of the dispersion and lifetime of both incoming and outgoing polaritons allows an excellent quantitative description of the results. This high-resolution Brillouin study opens different perspectives for the study of ground and excited polariton states and their dynamics in confined systems.
\end{abstract}

Long after its introduction for bulk semiconductors, ${ }^{1}$ the concept of a coupled exciton-photon polariton remains an important generic problem in condensed-matter physics. It has witnessed an actuality following the introduction of the concept of photon cavity polaritons ${ }^{2}$ and, more recently, with the observation of spontaneous coherence of polaritons with mechanisms which can be interpreted as nonequilibrium analogs of Bose-Einstein condensation. ${ }^{3}$ Resonant Brillouin scattering provided the first determination of the polariton dispersion in bulk semiconductors, assuming negligible damping. ${ }^{4}$ In the standard picture, the polariton at the incoming energy is scattered into a second polariton at the outgoing energy with the emission (Stokes, ST) or the absorption (antiStokes, AS) of an acoustic phonon whose energy and wave vector are obtained from the energy and wave-vector conservation during the scattering process. The Brillouin shifts measured at different incoming energies thus directly reflect the polariton dispersion, as reported in several bulk semiconductors. ${ }^{4}$ More recently, the relevance of the polaritonic picture to describe light scattering by cavity polaritons ${ }^{5}$ has been demonstrated. When finite exciton or photon lifetimes are taken into account, polariton damping is expected to emerge. Lifetime effects on Brillouin $^{6,7}$ and Raman ${ }^{8,9}$ resonant cross sections have been addressed. Up to now, the more direct influence of finite lifetimes on the Brillouin linewidth has, however, escaped observation because of the limited resolution of Raman setups and lasers as compared to lifetime-induced bulk polariton linewidths. Based on this fact, it has been also claimed ${ }^{10}$ that, with pure photons being the true initial and final states of the Brillouin scattering event, the polariton lifetime should not enter into the scattering process. Moreover, additional complications appear in the polariton picture in bulk materials because of the exciton energy dispersion: Two polariton branches coexist at the same energy in some cases and, to the best of our knowledge, the conversion of a single photon outside the sample into these multiple branches has not been completely understood. ${ }^{11}$ We show here that these issues can be conclusively addressed using high-resolution Brillouin studies of high-quality GaAs/AlAs multiquantum wells (MQWs). We emphasize that (a) the availability of unique acoustic branches due to the folding of acoustic branches by superperiodicity ${ }^{12}$ opens different scattering channels for the polaritons and different experimental access to the intrinsic properties of exciton polaritons in modulated semiconductors, and (b) the absence of dispersion of MQW excitons along the growth axis ${ }^{13}$ allows a full quantitative description of scattering events between uniquely defined polariton branches. In particular, we observed clear variations of the Brillouin linewidths, giving a unique access to the polariton damping in MQWs.

Previous Brillouin scattering reports on GaAs/AlAs multilayers focused on the emergence, at strong excitonic resonances, of broadband contributions to the Brillouin signal from isolated quantum wells due to the fluctuations of well widths from well to well. ${ }^{7,14}$ Based on a comparison between the exciton resonance profiles of folded acoustic lines and the broadband contributions, the inhomogeneous and homogeneous parts of the exciton linewidth and their variations with temperature have been determined independently in superlattices in which the inhomogeneous part is dominant. Several photoluminescence and photoluminescence excitation (PLE) studies have been devoted to the analysis of the exciton lifetime, mostly in single quantum wells. ${ }^{15-17}$ Based on measurements, as a function of well width and temperature, inhomogeneous contributions due to in-plane width fluctuations and homogeneous contributions, either independent (spin flip, impurity scattering) or dependent of the temperature (acoustic and optical phonon scattering), can be determined. In the best quality samples, photoluminescence half widths at half maximum (HWHM) as low as $0.15 \mathrm{meV}$ have been obtained in 15-nm-thick quantum wells at liquid-helium temperature. Only small increases due to acoustic phonon scattering have been measured up to liquid nitrogen. Polaritonic effects have not been considered in these previous reports either due to large inhomogeneous linewidths or due to the absence of 
periodicity in single quantum wells. We report here resonant Brillouin results on a multiquantum well with quality close to the best single quantum wells described in the literature. This allows us to demonstrate polaritonic effects in the Brillouin line resonant dispersion and linewidth and to obtain quantitative access to the polaritonic damping in these conditions.

We have performed resonant Brillouin scattering on a 40period multiquantum well with $17.1 \mathrm{~nm}$ of GaAs and $7.5 \mathrm{~nm}$ of AlAs in each period. The quality of this MQW is exceptional, as demonstrated by a HWHM at $80 \mathrm{~K}$ of the exciton line of as low as $0.3 \mathrm{meV}$ in PLE and $0.5 \mathrm{meV}$ in photoluminescence, and a Stokes shift comparable to the PLE linewidth. The Brillouin experiments have been performed with a Coherent monolithic block resonator (MBR) single-mode Ti-Sa tunable ring laser with a wavelength stability of better than $0.01 \mathrm{~cm}^{-1}$. Resonance profiles have been measured close to different confined exciton levels and we focus here on the E1-HH1 transition between the lowest conduction electron state and the one of the heavy hole. The resonance curve has been followed using laser energy steps close to $25 \mu \mathrm{eV}\left(0.2 \mathrm{~cm}^{-1}\right)$. The light emitted by the sample, including excitonic luminescence, Brillouin scattering, and the peak at the laser energy, has been dispersed by a Dilor $X Y$ triple Raman spectrometer in additive mode and recorded with a nitrogen-cooled CCD multichannel detector. The experimental resolution is close to $0.2 \mathrm{~cm}^{-1}$. We discuss here the experimental results obtained at $80 \mathrm{~K}$. At higher temperatures, all intensities decrease, imposing longer experimental times to access the same physics. At lower temperatures, the excitonic luminescence increases strongly and the Brillouin spectra exhibits qualitative changes, reflecting different physics, possibly due to an increasing polariton localization, which is beyond the scope of this Rapid Communication. Thanks to the large intensity of the narrow Brillouin peaks, we have been able to record the different scattered lines in the same CCD window without having to filter out the laser or the exciton luminescence.

In Fig. 1 we show the Brillouin scattering spectra measured at a few different incident energies across the excitonic transition, plotted in Fig. 1(a) as a function of the absolute scattered energy given in $\mathrm{eV}$ and in Fig. 1(b) relative to the laser energy (Raman shift in $\mathrm{cm}^{-1}$ ). The Brillouin lines, associated with longitudinal acoustic phonons (LA), with a shift close to $1.3 \mathrm{~cm}^{-1}$, and to the lowest folded acoustic phonons (FLA-1 and FLA +1 ), with shifts at $\sim 5$ and $8 \mathrm{~cm}^{-1}$, respectively, are observed on each side of the most intense laser line, respectively corresponding to Stokes and anti-Stokes processes, and on top of the excitonic luminescence. In Fig. 1(a), one clearly sees the large variations of the intensity, plotted in logarithmic scale, and of the linewidth of the different peaks. In the spectrum in gray, with laser energy close to the exciton transition energy $(1.5246 \mathrm{eV})$, all peaks are significantly broadened. In the spectra in light gray and dark gray, the FLA lines with absolute energies close to the exciton transition are significantly broadened as well. In Fig. 1(b), the variation of the line shifts with the incident energy becomes apparent, going toward opposite directions for the LA line and the FLA-1 line, respectively. We have performed a detailed line-shape analysis in order to systematically extract the positions and the widths of the different lines in the spectra, after having removed the background due to the excitonic
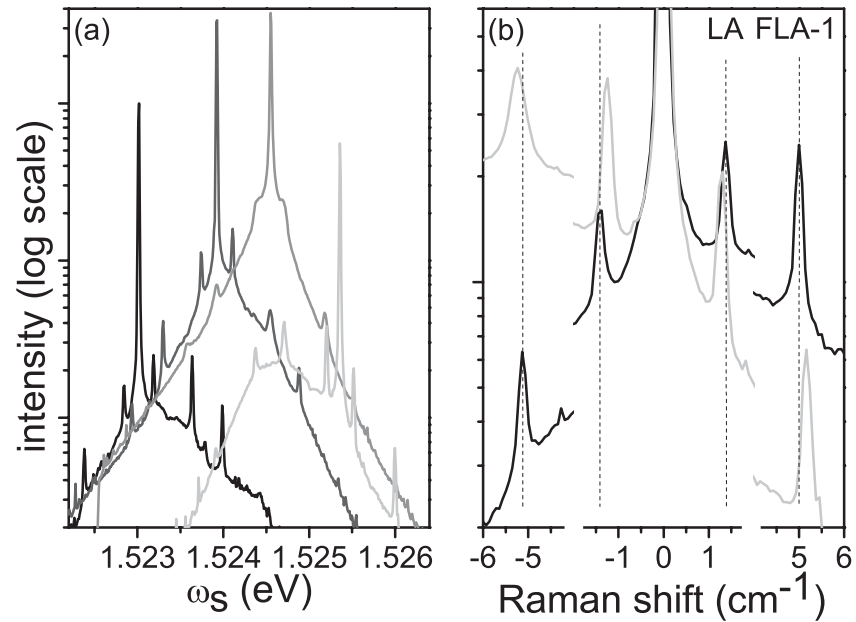

FIG. 1. (a) Resonant Brillouin spectra at four different incident laser energies across the E1-HH1 excitonic resonance. In each spectrum the most intense line is at the laser energy and the weaker lines are on each side at the inelastically scattered energies. In (b) the two curves with the smallest and the largest incident energies in the left-hand panel are shown with an energy scale relative to the laser position (Raman shift). The vertical lines highlight the change in the Raman shifts across the resonance.

luminescence. Thanks to the coexistence of the laser line and the Brillouin peaks in the same spectra, very high accuracy has been obtained for the Brillouin shift, the distance between the inelastic and the elastic lines.

We have deduced very systematic variations as a function of the incident energy, with a very high signal-to-noise ratio. As shown in Fig. 2, these variations exhibit systematic trends when the different lines, and the Stokes and anti-Stokes components for each one, are compared. For the LA mode [Fig. 2(a)], the shifts display an oscillation close to the exciton resonance, with a typical amplitude of $0.2 \mathrm{~cm}^{-1}$, while the widths display a maximum at the same position. Outside the resonance the linewidth is dominated by the resolution of the spectrometer, amounting to $0.2 \mathrm{~cm}^{-1}$, while its peak value is of the order of $0.5 \mathrm{~cm}^{-1}$. One should note that the intrinsic acoustic phonon lifetime is extremely long and has a negligible influence on the linewidth. The results for the Stokes and anti-Stokes components are very similar except for a small energy shift in the features. In the case of the FLA modes [Figs. 2(b) and 2(c)], two successive oscillations of the shifts are observed both in the Stokes and the anti-Stokes components. One of these oscillations is at the same energy for both components while the second one appears at higher (lower) energy for the Stokes (anti-Stokes) component. The features for the width variations display very similar trends: two peaks in Stokes components and two others in the anti-Stokes components. Each peak displays a HWHM of the order of $0.15 \mathrm{meV}$, which is significantly smaller than the corresponding values for the photoluminescence and the PLE results. Again, a common peak appears at the energy of the exciton both in Stokes and the anti-Stokes components, while the second one is shifted symmetrically, at higher (lower) energy as compared to the exciton position in the anti-Stokes (Stokes) component. 


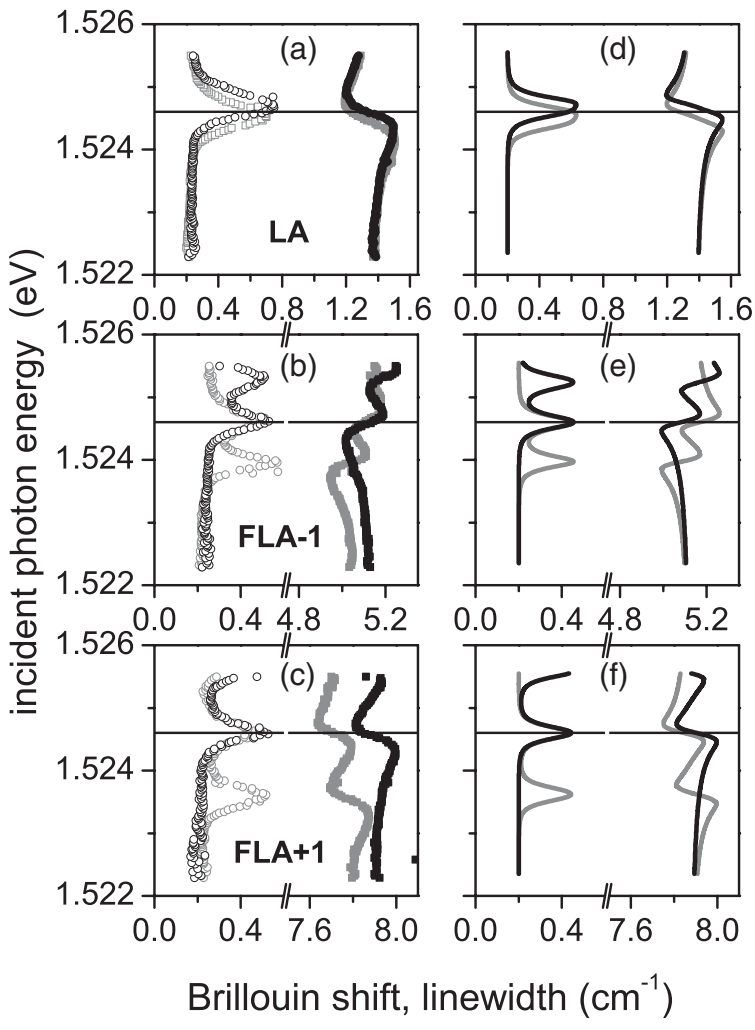

FIG. 2. (a)-(c) Variation with the incident energy of the Brillouin shift (solid squares) and the Brillouin linewidth (open circles) deduced from the experimental spectra corresponding to longitudinal acoustic phonons [LA, (a)], and first folded longitudinal acoustic phonons [FLA-1, (b) and FLA + 1, (c)]. In each panel, the data are represented for the Stokes (black labels) and the anti-Stokes (gray labels) lines. (d)-(f) Calculated values of the same quantities as in (a)-(c), respectively, according to the model described in the text. The horizontal line in all panels indicates the fitted exciton energy (incoming resonance).

This behavior can be attributed to the combination of incoming and outgoing resonances with the damped exciton polariton in the MQW. The eigenvalue problem for interacting photons and excitons has been solved for bulk materials by Hopfield, ${ }^{1}$ introducing coupled excitations called exciton polaritons. When the nonradiative exciton damping $\gamma$ is included, the relation between the polariton energy $\omega$ and wave vector $k$ is written as

$$
\frac{c^{2} k^{2}}{\omega^{2}}=\varepsilon_{0}\left(1+\frac{\omega_{P}}{\omega_{X}-\omega-i \gamma}\right),
$$

in which $\varepsilon_{0}$ is the background dielectric constant, $\hbar \omega_{X}$ the exciton energy, and $\hbar \omega_{P}$ reflects the strength of the exciton-photon coupling. In the absence of damping, this equation leads to the existence of two branches of polaritons. The dispersion can be described by two parameters: (a) the longitudinal transverse splitting $\omega_{\mathrm{LT}}$, i.e., the width of the gap between the lower and the upper polariton branches in which no states exist due to the polariton coupling, and (b) the Rabi splitting $\omega_{R}$, i.e., the minimum separation between the two branches at a given wave vector. For small exciton-photon coupling, $\omega_{\mathrm{LT}} \simeq \omega_{P}$ is much smaller than $\omega_{R} \simeq \sqrt{2 \omega_{P} \omega_{X}}$. Using standard GaAs parameters, ${ }^{4} \omega_{\mathrm{LT}}=0.086 \mathrm{meV}$ and $\omega_{R}=16 \mathrm{meV}$.

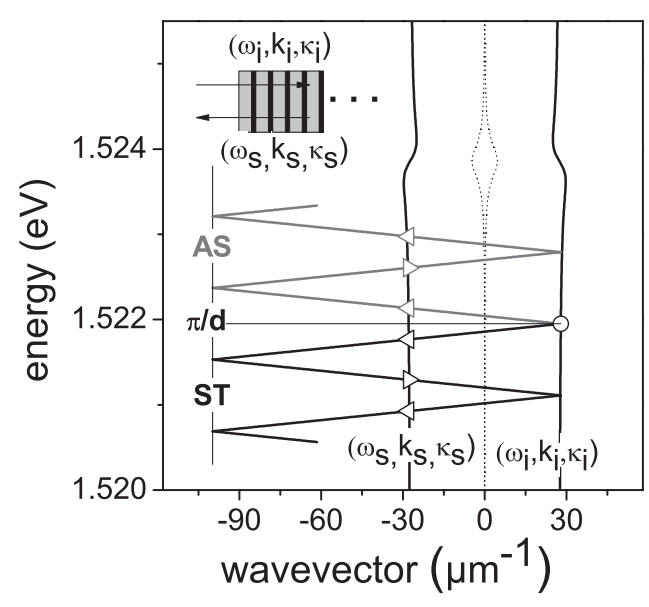

FIG. 3. Schematic description of the resonant Brillouin process between an ingoing polariton (black circle) and outgoing polaritons for Stokes (ST, black triangles) and anti-Stokes (AS, gray triangles) contributions. The solid (dashed) black lines show the real part $\left(k_{s}, \omega_{s}\right)$ and the imaginary part $\left(\kappa_{s}, \omega_{s}\right)$ of the incident and scattered polariton dispersion curves calculated with typical parameters for the studied sample. The geometry of the experimental configuration is schematized in the inset. From the incoming polariton, folded phonon dispersions are traced on the positive energy side (AS, gray lines) and the negative side (ST, black lines), crossing the scattered polariton dispersion at successive points associated with the scattering by LA and folded LA phonons.

The polariton description has been extended successfully to the case of excitons in quantum wells and superlattices. ${ }^{13,18}$ It has been demonstrated that Eq. (1) applies, using an effective exciton-photon coupling, to superlattices in the long-wavelength limit, which applies to the optical properties studied here. Moreover, the absence of dispersion for the MQW exciton along the growth direction greatly simplifies the polariton model based on Eq. (1) as compared to the bulk situation. A single damped polariton branch then exists at any energy, and its dispersion and damping can be described introducing a complex wave vector $k+i \kappa$ in Eq. (1). In Fig. 3 we show the polariton dispersion by two curves (thick and dotted lines) corresponding to the dispersion of the real part and the imaginary part of the wave vector, respectively. We used a damping of $0.15 \mathrm{meV}$, a value corresponding to the experiments reported here. This damping is significantly larger than the longitudinal transverse splitting, thus making it irrelevant. Nevertheless, the concept of the polariton remains essential as this damping is much smaller than the Rabi gap, leading to a significant modification of the dispersion around the exciton energy due to the polariton coupling.

In Fig. 3, the scattering processes between an incident polariton $\left(\omega_{i}, k_{i}, \kappa_{i}\right.$, black open circle $)$ and a scattered polariton $\left(\omega_{s}, k_{s}, \kappa_{s}\right.$, open black gray and gray triangles) by acoustic phonons is described schematically. The folded dispersion with the LA branch and the FLA $\pm n$ ones at energies $v k$ and $v\left(n \frac{2 \pi}{d} \pm k\right)$, respectively ( $d$ is the period of the superlattice and $v$ the sound velocity) is inserted with the wave-vector-energy origin at the incident polariton state. The dispersion in gray at higher energies corresponds to the absorption of a phonon (anti-Stokes process), while the black 
curve at lower energies corresponds to the Stokes emission process. The energy of the scattered polaritons is given by the crossing points between the folded phonon dispersion and the scattered polariton dispersion. Variations in the acoustic phonon energies and linewidths are expected when either the incident or the scattered polariton energies coincide with the exciton one.

At the incoming resonance, when the laser energy coincides with the exciton one, one expects a common feature in all the experimental traces, which is the case in our data for $1.5246 \mathrm{eV}$. The outgoing resonance is shifted from the incoming one by the Brillouin shift, which is positive or negative whenever the Stokes or the anti-Stokes component is considered. In the case of the LA line [Fig. 2(a)], the Brillouin shift is too small to allow for a clear separation of the two resonance channels, and a single resonance is observed at a slightly different position for the Stokes and the anti-Stokes components. The amplitude of the oscillation and the linewidth peak value are larger than for the FLA lines because they reflect the combination of two resonances. In the case of the FLA lines [Figs. 2(b) and 2(c)], the experimental observation of three identical oscillations in the energy shift and three identical peaks in the line broadening, one being common to the Stokes and the anti-Stokes components and the other ones symmetrically shifted toward higher or lower energy in ST and AS, respectively, qualitatively demonstrate the successive emergence of separate outgoing and ingoing resonances when the incoming energy is varied.

Using the discrete damped polariton dispersion given by Eq. (1), we calculated the Brillouin shifts, fulfilling both the conservation of the energy and the wave vector in a scattering event with an acoustic phonon, both in the Stokes and anti-Stokes channels. The result of this calculation is shown in the right-hand panels of Fig. 2, with the same colors as used in the left-hand panel for experimental results. The full width at half maximum is obtained from the expression $2 v\left(\kappa_{i}+\kappa_{s}\right)$. The experimental resolution $\left(0.2 \mathrm{~cm}^{-1}\right)$ is taken into account by a convolution formula strictly valid for Gaussian profiles only. We have taken the parameters given for bulk GaAs in Ref. 4: a background dielectric constant equal to 12.55 and a longitudinal transverse splitting of $0.086 \mathrm{meV}$. The exciton energy has been fitted to $1.5246 \mathrm{eV}$ and the lifetime parameter $\gamma$ to $0.15 \pm 0.02 \mathrm{meV}$. With these parameters, the width and the amplitude of the structures in the energy and linewidth resonance profiles are excellently reproduced. The peak linewidth is slightly underestimated by an amount within the uncertainty in the line-shape fitting and the simplified treatment of the instrumental broadening.
The bulk $\omega_{\mathrm{LT}}$ value allows a good description of the experimental results as the variation due to confinement is expected to be small for such a wide quantum well. ${ }^{19}$ Some discrepancy remains in the background variations of the line energies, negligible for the LA peaks, and of increasing magnitude with the energy shift. This is likely to be due to additional contributions to the dielectric constant of higher-energy band-to-band transitions, not included in the model.

In conclusion, we have observed very clear evidence of the polaritonic coupling in light scattering by acoustic and folded acoustic phonons in a multiquantum well when the polariton damping is larger than the longitudinal transverse splitting but significantly smaller that the Rabi splitting. Thanks to the combination of a specifically designed high-resolution setup, a multiquantum well with much thicker layers than in previous studies, and an optimization of the experimental temperature, exceptionally clear variations of the position and the width of Brillouin lines have been demonstrated when the laser energy satisfies either the incoming or the outgoing resonance. Further information is obtained through the observation of similar consistent effects on different folded acoustic modes. Moreover, the higher energy of these modes allows to resolve the separate contributions to Brillouin shifts and lifetimes from the incoming and outgoing polariton channels, which, to the best of our knowledge, has been impossible when the standard acoustic modes are the only ones present in bulk materials. A quantitative description is obtained in a damped polariton picture provided a broadening of $0.15 \mathrm{meV}$ is included, a value significantly smaller than the exciton linewidth measured in PLE and comparable to the best single quantum-well exciton HWHMs reported in the literature. ${ }^{15-17}$ This suggests that light scattering gives access to information on the damping mechanisms in multiquantum wells, possibly excluding the inhomogeneous broadening from well to well owing to the delocalized nature of the probing acoustic phonons. We also believe that the approach presented here is a highly valuable tool to address fundamental problems related to polariton dispersion and damping in the presence of photonic confinement, in particular, for Bragg superlattices, an interesting alternative to photon cavities in which the modulation period fits the photon wavelength at the energy of confined excitons. ${ }^{18,20}$

This work is a part of the International French Argentinian Nanoscience Laboratory, LIFAN. We would like to thank very much J. Bloch for several discussions on the polariton properties.
${ }^{1}$ J. J. Hopfield, Phys. Rev. 112, 1555 (1958).

${ }^{2}$ C. Weisbuch, M. Nishioka, A. Ishikawa, and Y. Arakawa, Phys. Rev. Lett. 69, 3314 (1992).

${ }^{3}$ J. Kasprzak, M. Richard, S. Kundermann, A. Baas, P. Jeambrun, J. M. J. Keeling, F. M. Marchetti, M. H. Szymanska, R. André, J. L. Staehli, V. Savona, P. B. Littlewood, B. Deveaud, and Le Si Dang, Nature (London) 443, 409 (2006).
${ }^{4}$ C. Weisbuch and R. G. Ulbrich, in Light Scattering in Solids III, edited by M. Cardona and G. Güntherodt (Springer, Berlin, 1982), p. 207.

${ }^{5}$ A. Fainstein, B. Jusserand, and V. Thierry-Mieg, Phys. Rev. Lett. 78, 1576 (1997).

${ }^{6}$ S. Adachi and C. Hamaguchi, Phys. Rev. B 19, 938 (1979). 
${ }^{7}$ T. Ruf, V. I. Belitsky, J. Spitzer, V. F. Sapega, M. Cardona, and K. Ploog, Phys. Rev. Lett. 71, 3035 (1993).

${ }^{8}$ A. Bruchhausen, A. Fainstein, B. Jusserand, and R. André, Phys. Rev. B 68, 205326 (2003).

${ }^{9}$ A. Bruchhausen, L. M. Leon Hilario, A. A. Aligia, A. M. Lobos, A. Fainstein, B. Jusserand, and R. André, Phys. Rev. B 78, 125326 (2008).

${ }^{10}$ R. Zeyher, C. S. Ting, and J. L. Birman, Phys. Rev. B 10, 1725 (1974).

${ }^{11}$ B. Bendow, in Electronic Structure of Noble Metals and Polariton-Mediated Light Scattering (Springer, Berlin, 1978), p. 69.

${ }^{12} \mathrm{~B}$. Jusserand and M. Cardona, in Light Scattering in Solids $V$, edited by M. Cardona and G. Güntherodt (Springer, Berlin, 1989), p. 49.

${ }^{13}$ L. C. Andreani, Phys. Lett. A 192, 99 (1994).
${ }^{14}$ D. N. Mirlin, I. A. Merkulov, V. I. Perel', I. I. Reshina, A. A. Sirenko, and R. Planel, Solid State Commun. 84, 1093 (1992).

${ }^{15}$ Vivek Srinivas, John Hryniewicz, Yung Jui Chen, and Colin E. C. Wood, Phys. Rev. B 46, 10193 (1992).

${ }^{16}$ J. Martinez-Pastor, A. Vinattieri, L. Carraresi, M. Colocci, Ph. Roussignol, and G. Weimann, Phys. Rev. B 47, 10456 (1993).

${ }^{17}$ D. Gammon, S. Rudin, T. L. Reinecke, D. S. Katzer, and C. S. Kyono, Phys. Rev. B 51, 16785 (1995).

${ }^{18}$ E. L. Ivchenko, Fiz. Tverd. Tela (Leningrad) 33, 2388 (1991) [Sov. Phys. Solid State 33, 1344 (1991)]; E. L. Ivchenko, A. I. Nesvizhskii, and S. Jorda, Fiz. Tverd. Tela (Leningrad) 36, 2118 (1994) [Sov. Phys. Solid State 36, 1156 (1994)].

${ }^{19}$ L. C. Andreani and A. Pasquarello, Phys. Rev. B 42, 8928 (1990).

${ }^{20}$ Y. Merle d'Aubigné, A. Wasiela, H. Mariette, and T. Dietl, Phys. Rev. B 54, 14003 (1996). 